\title{
The Time Course of Visual Processing: From Early Perception to Decision-Making
}

\author{
Rufin VanRullen and Simon J. Thorpe
}

\begin{abstract}
Experiments investigating the mechanisms involved in visual processing often fail to separate low-level encoding mechanisms from higher-level behaviorally relevant ones. Using an alternating dual-task event-related potential (ERP) experimental paradigm (animals or vehicles categorization) where targets of one task are intermixed among distractors of the other, we show that visual categorization of a natural scene
\end{abstract}

How do we perceive and understand a natural scene? What are the mechanisms involved in the extraction of its meaning, and how do they temporally relate to each other? Current theories of visual processing suggest a distinction between two different mechanisms: a perceptual process extracting information about different properties of the visual input, followed by a higher-level decision process evaluating the relevance of this visual information, in terms of the goals and expectations of the subject, in order to prepare and generate the appropriate behavioral response (Romo \& Salinas, 1999; Schall \& Thompson, 1999; Shadlen \& Newsome, 1996). How these two mechanisms can be dissociated in time and space is a fundamental issue that is not easy to address (Nichols \& Newsome, 1999; Schall \& Thompson, 1999). In humans in particular, using reaction times as a dependent variable precludes the separation of the respective durations of perception, decision, and motor response stages (Luce, 1986).

Event-related potentials (ERPs), which can be recorded even in the absence of a behavioral response, have been shown to reflect high-level properties of the visual stimulus such as its identity or category after roughly 150 msec (Mouchetant-Rostaing, Giard, Bentin, Augera, \& Pernier, 2000; Allison, Puce, Spencer, \& McCarthy, 1999; Schendan, Ganis, \& Kutas, 1998; Thorpe, Fize, \& Marlot, 1996; Bötzel, \& Schulze, Stodieck, 1995). This holds for faces (Jeffreys, 1996) as well as other objects (Rossion et al., 2000). More recently, magnetoencephalography (MEG) has demonstrated a similar pattern of results (Halgren, Raij, Marinkovic, Jousmäki, \& Hari, 2000). Likewise, experiments using electrophy-

Centre de Recherche Cerveau et Cognition, France involves different mechanisms with different time courses: a perceptual, task-independent mechanism, followed by a taskrelated, category-independent process. Although average ERP responses reflect the visual category of the stimulus shortly after visual processing has begun (e.g. 75-80 msec), this difference is not correlated with the subject's behavior until $150 \mathrm{msec}$ poststimulus.

siological recordings in monkeys have reported neural responses highly selective to a specific visual category even before 100 msec (Vogels, 1999; Oram \& Perrett, 1992; Perrett, Rolls, \& Caan, 1982). A number of recent ERP studies in humans (Mouchetant-Rostaing et al., 2000; Debruille, Guillem, \& Renault, 1998; George, Jemel, Fiori, \& Renault, 1997; Seeck et al., 1997) seem to suggest, however, that high-level properties of the visual stimulus could be extracted much faster, sometimes reporting "face recognition" (Seeck et al., 1997) or "face gender discrimination" (Mouchetant-Rostaing et al., 2000) effects as early as 50 msec poststimulus.

However, the fact that neural activity varies with the properties of the visual input is not sufficient to conclude that the subject can actively recognize the identity of the categories involved. For example, it could simply reflect differences in the low-level properties of the visual stimuli, differences that are difficult if not impossible to investigate systematically.

Here we present an alternating dual-task paradigm ERP experiment that allowed us to compare the processing of the same visual category having different task-related status, and conversely, different visual categories having the same behavioral status. We were able to dissociate (i) a low-level sensory analysis activity and (ii) a high-level task-related mechanism that was clearly independent of the sensory properties of the stimulus.

\section{RESULTS}

\section{Animal and Vehicle Tasks}

In one task, replicating the go/no-go experiment from Thorpe et al. (1996), 16 subjects were seated in front of a 
computer screen and had to release a button if the natural photograph that was flashed for only $20 \mathrm{msec}$ contained an animal. ERPs were recorded concurrently on 32 electrode sites. In the second task, the same 16 subjects were asked to respond on images belonging to the target category "means of transport." In each task, half of the nontarget images belonged to the target category of the other task (i.e., vehicles in the animal task, animals in the vehicle task). The other half were distractor scenes that contained no animal or vehicle. To compare the two tasks under the same conditions, 10 series of 96 trials for each task were performed in alternation (20 in total). In different blocks, images of one particular category could be treated either as targets or as nontargets, so as to allow comparison of the processing of the same visual category in target and nontarget trials. Furthermore, half of the vehicle scenes were pictures of cars, in order to perform intracategory comparisons. Images in each category were chosen to be as varied as possible. Figure 1 shows two examples of image sequences that were viewed by one subject. Animal images could contain mammals but also birds, fish, or insects. Vehicle images included cars as well as trucks, trains, boats, airplanes, helicopters, or hot air balloons. The size, number, and position of the targets in the scenes were unpredictable. There was also a very wide range of distractor scenes that could contain landscapes, trees, roads with buildings, man-made objects, etc.

Behavioral results have been presented elsewhere (VanRullen \& Thorpe, in press) and showed no difference in performance between the animal and vehicle categorization tasks. Percentages correct are around 94\% and median reaction times slightly above $350 \mathrm{msec}$. We also presented evidence that categorization could be
Figure 1. Two sequences of 12 images presented in the animal and the vehicle categorization tasks, with reaction times from the fastest subject (median reaction times $288 \mathrm{msec}$ in the animal task, $293 \mathrm{msec}$ in the vehicle task, percentages correct $92.7 \%$ and $92.6 \%$, respectively).

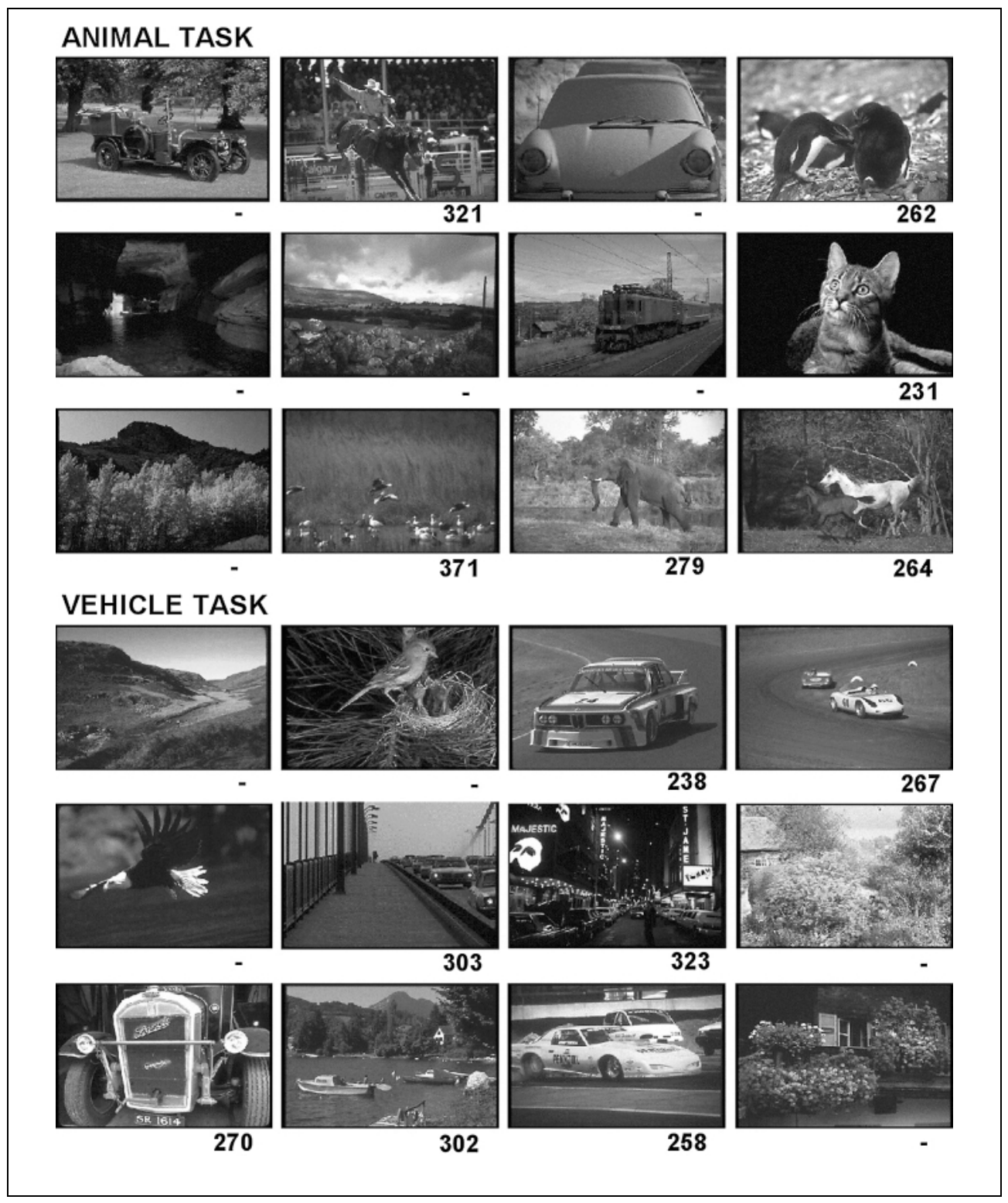




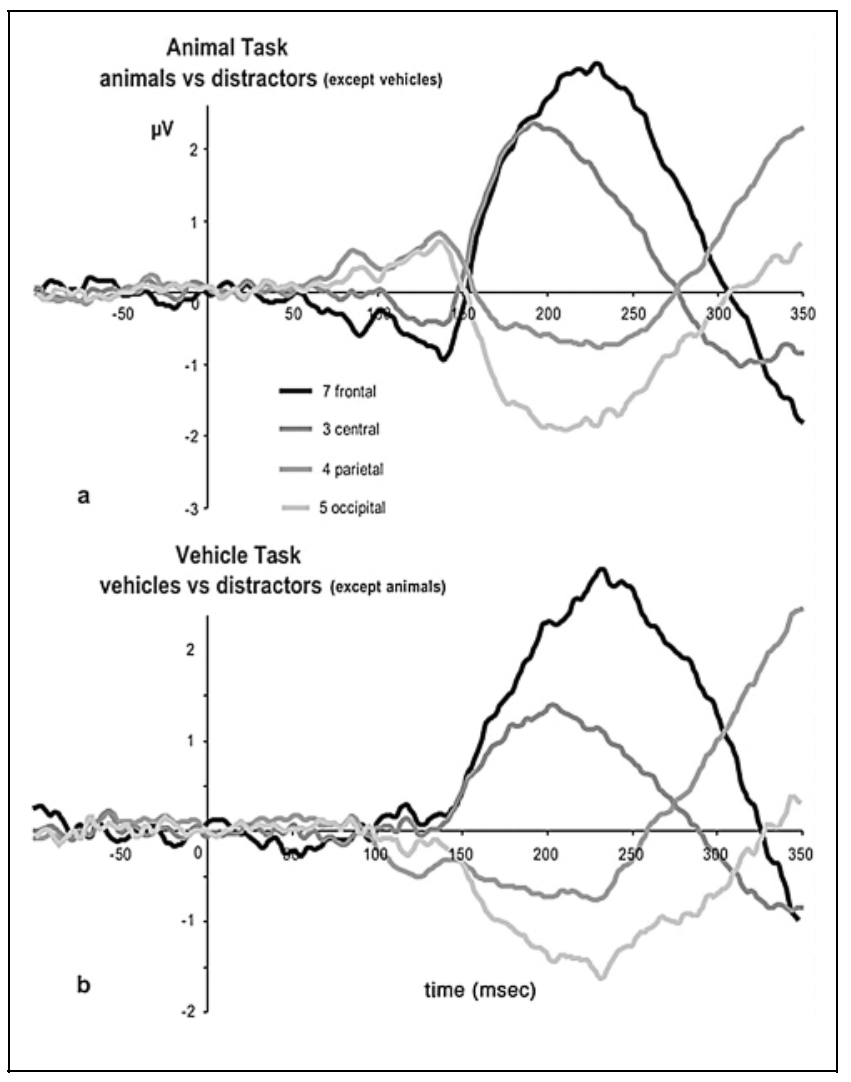

Figure 2. Difference waveforms between target and distractor images in the two (animal and vehicle) categorization tasks (not including the nontarget images that belong to the target category of the other task). Grand-average over all 16 subjects. Each panel shows the difference waveforms for the seven frontal, three central, four parietal, and five occipital electrodes. performed above chance in less than 250 msec, a surprisingly short value which gives an upper limit to the duration of perceptual processes and the beginning of the subject's decision. ERPs can strengthen these constraints and provide important insights about the time course of the underlying visual processing.

In each task, we find a strong differential activity between targets and distractors that can best be seen on frontal electrode sites after $150 \mathrm{msec}$ (see black lines, Figure 2), as had been reported for an animal categorization task (Thorpe et al., 1996). At the same moment, there are also clear differential responses at virtually all recording sites (central, parietal, occipital), thus stressing the magnitude of the effect. Furthermore, these previous results are reproduced here for a task involving a different target category. It is important to stress that "means of transport" being a clearly artificial category, there does not seem to be any advantage of processing natural, biologically relevant visual stimuli.

In addition to this strong differential activity between targets and distractors starting around $150 \mathrm{msec}$, Figure 2 also illustrates an earlier, weaker differential activity arising roughly $75 \mathrm{msec}$ after stimulus presentation. This effect, which was not reported in the previous study by Thorpe et al. (1996), can certainly be attributed to changes made in the experimental protocol. In particular, the angular size of images was twice the size used previously, which might increase the signal-to-noise ratio. Furthermore, ERPs were recorded here using an array of 32 average-referenced electrodes, instead of 20 linked-ears-referenced electrodes in the previous study.
Figure 3. Effects of the visual category. Each panel shows the differential activity between two visually defined categories. The taskrelated status (target or nontarget) is equally represented in each category. For simplicity, intersubjects standard error of the mean (SEM) is plotted only for parietal electrodes (dotted gray curves). (a) Animals versus vehicles on the seven frontal, three central, four parietal, and five occipital electrode groups. (b-d) Difference waveforms on parietal electrodes. (b) Animals versus cars. (c) Animals versus noncar vehicles. (d) Cars versus noncar vehicles. Statistical $t$ tests $(d f=15)$ were performed for each of these difference waveforms between categories on parietal electrodes where the effect is best seen. At the $p<.01$ level, the potentials evoked by animal images significantly diverge from the vehicle-evoked potentials at $77 *$ msec poststimulus and for 82 consecutive samples (panel a). More specifically, animal and car trials diverge after $92 *$ msec $(+74$ samples, panel b) and the statistical difference between animal and noncar vehicle trials appears at 76* msec ( +25 samples, panel c). Finally, the differential activity between cars and noncar vehicles pictures significantly differs from zero after 81 msec and for four consecutive samples, after which the sign of the difference is reversed, and reaches significance again at $98^{*} \mathrm{msec}(+91 \mathrm{samples}$, panel d). The sign * indicates significance according to the criterion of Rugg et al. (1995), modified for stronger precision: 15 consecutive $t$ test values below the $p<.01$ level. 
In regard to the distinction between perceptual and higher-level task-related decision mechanisms mentioned above, how should each of these two differential activities be interpreted? Is it the correlate of low-level, systematic visual differences between the target and distractor categories involved, or does it reflect the subject's decision that the target category has been detected? The next sections will attempt to separate the causes of these two differential effects.

\section{Visual Category Effects}

The different visual categories involved (animals, vehicles, further separated as cars and other vehicles) can be compared to one another in a task-independent manner. For each subject, we averaged the waveforms of images belonging to the same category independent of their target/nontarget status (by weighing each taskrelated status according to the number of corresponding trials), then compared these category-specific waveforms to one another. The difference waveforms are presented in Figure 3. The differential activities between any two visual categories appear to diverge from zero after $75-80 \mathrm{msec}$, which is confirmed by the statistical $t$ tests performed on parietal electrodes (see caption on Figure 3 for details). These differences are totally independent of the task, and on the status of the images in these tasks (target and nontarget trials are equally represented in each category-specific waveform). In particular, objects in the car and the noncar vehicle categories have exactly the same status in both tasks: targets in the vehicle categorization task, nontargets in the animal categorization task. In this case, the difference (Figure 3d) can only be attributed to intrinsic visual properties of these categories, and not to differences in the task being performed.

\section{Task-Related Effects}

What is the specific activity induced by the categorization task at hand, that is, the difference between the processing of targets versus nontargets? To answer this question, we can group together all the waveforms corresponding to different categories of stimulus when they were targets, and compare the result with the result of grouping together waveforms for the same categories of stimulus, but when they were treated as nontargets. Figure $4 \mathrm{a}$ shows the differential waveforms of the animals and vehicles grouped together, between the "target" and "nontarget" conditions. Figure 4b-e shows the same difference separately for each category, on frontal electrode sites. The earliest statistically significant effects for these differential activities are obtained between 156 and $180 \mathrm{msec}$ (see caption on Figure 4 for details). This difference in processing between target and nontarget trials, starting at $150 \mathrm{msec}$ poststimulus and reaching significance around $160 \mathrm{msec}$, clearly constitutes the equivalent in our dual-task paradigm of the difference between target and distractor trials reported in a previous study (Thorpe et al., 1996). However, the effect that we demonstrate here is totally independent of the visual category involved, because the difference is ob-

Figure 4. Effects of the categorization task. Each panel shows the differential activity between target and nontarget trials for a visually defined category. In each condition (target and nontarget), the visual categories involved are represented equally. For simplicity, SEM is plotted only for frontal electrodes (dotted black curves). For readability, the $y$ axis represents electrical activity on a different scale than Figure 3. (a) Targets versus nontargets in both tasks (animals and vehicles). (b-e) Differential activity on frontal electrodes. (b) Animal category, targets versus nontargets. (c) Vehicle category, targets versus nontargets.

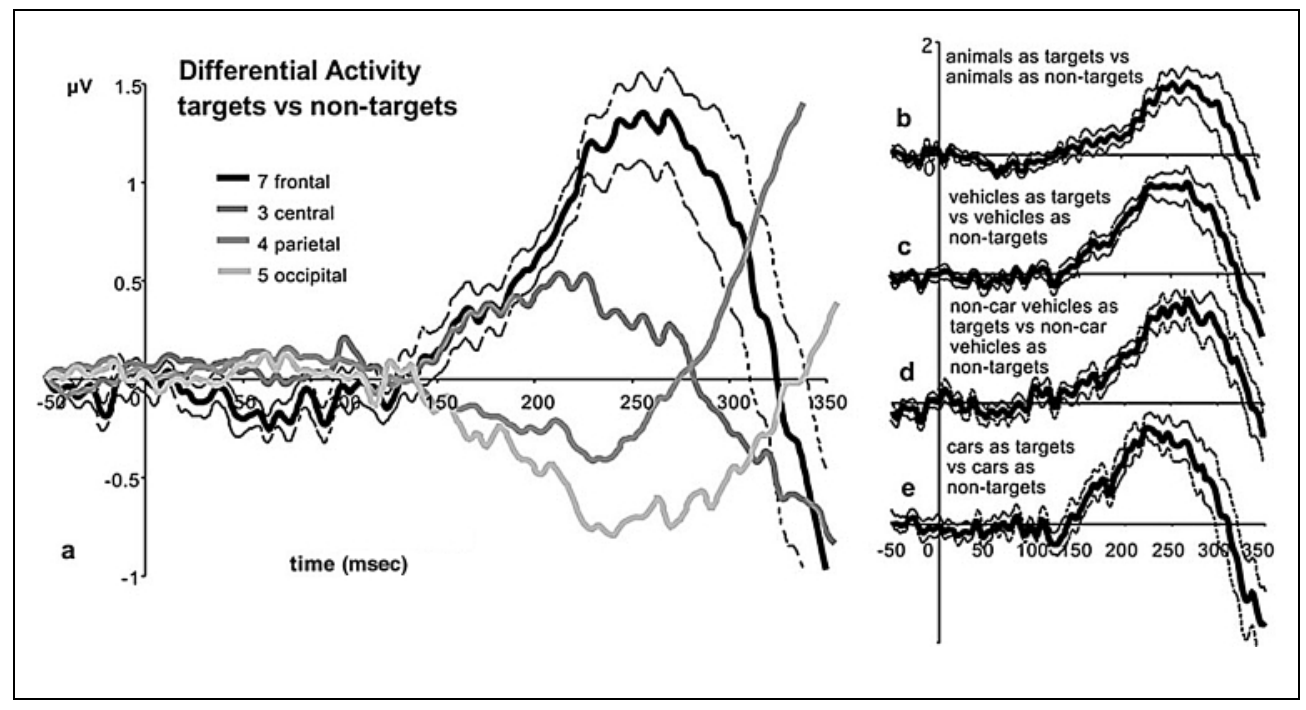
(d) Noncar vehicle category,

targets versus nontargets. (e) Car category, targets versus nontargets. For the difference shown in panel a, significance at the $p<.01$ level $(t$ test, $d f$ $=15)$ is reached on frontal electrodes at $175 *$ msec $(+120$ samples) but as early as $156 *$ msec $(+77$ samples) on central electrodes. On frontal electrodes, significance at the $p<.01$ level is achieved at $212 *$ msec ( +72 samples) for the difference shown in panel b, at $164 \mathrm{msec}(+6$ samples) for vehicles as targets versus nontargets (panel c), and more specifically at $189 *$ msec (+114 samples) for other vehicles (panel d) and at 165 msec ( +4 samples) for cars (panel e). However, earlier significant effects are seen on central electrodes for the animal images (156* msec, $+25 \mathrm{samples})$ and on occipital electrodes for vehicles $(160 \mathrm{msec},+4$ samples $)$ and noncar vehicle images $(177 *$ msec, +139 samples $)$. The sign * indicates significance according to the modified Rugg et al. (1995) criterion, as in Figure 3. 
served even when comparing the ERPs to target and nontarget trials on images belonging to the same visual category. The underlying mechanism is thus only correlated with the subject's decision, rather than with any visual property of the stimulus. Note that no differential activity between targets and nontargets is observed before $150 \mathrm{msec}$, which implies that the earlier difference that we found at 75-80 msec does not carry any information about the task-related status (target or nontarget) of the visual stimulus.

\section{Two Separate Mechanisms}

We isolated two different mechanisms. The first is an early perceptual, category-dependent, task-independent process, starting at 75-80 msec after stimulus onset. The second is a task-related, category-independent mechanism clearly correlated with the subject's decision that a target was or was not present in the image. This latter effect is found to start after $150 \mathrm{msec}$. What specific neural structures are involved in these two separate processes? Given, on the one hand, the latency of the first perceptual activity (i.e., $75-80 \mathrm{msec}$, peaking at around $120 \mathrm{msec}$ ), and the specific requirements of the task, probably involving mechanisms such as visual features extraction (e.g., shape), this first differential activity could arise in extrastriate visual areas such as V2 or V4. Similar occipital regions have been found in a recent MEG study (Halgren et al., 2000) to respond differently to different categories of stimuli with latencies around $110 \mathrm{msec}$, namely, of the same order as the one found here. At first sight, it seems surprising that a differential activity carrying information about the visual category of the stimulus perceived could be supported by "early" extrastriate visual areas. But at virtually any stage in the visual system, the information being extracted is likely to be correlated with the visual input, and thus to differ from one visual category to the other; this does not imply that the identity of the stimulus is actively encoded in these regions, and that visual processing is already terminated.

On the other hand, when neural activity is correlated with the decision of the subject rather than with the visual input, it implies that sufficient processing has been done to allow target detection (Romo \& Salinas, 1999; Shadlen \& Newsome, 1996; Newsome, Britten, \& Movshon, 1989). The objects in the visual scene have therefore been recognized and categorized: such a process is likely to occur at a rather late stage in the ventral occipito-temporal processing stream. Occipitotemporal activations have already been reported using event-related fMRI methods for an animal/nonanimal categorization task (Fize et al., 2000). Intracranial recordings in humans have also shown that ventral occipito-temporal regions can be highly responsive to faces and other objects with latencies between 150 and 200 msec (Allison et al., 1999). fMRI experiments also report regions in the ventral occipito-temporal cortex of humans that are differentially activated for different categories (e.g., faces, houses, chairs) of visual stimulus (Ishai, Ungerleider, Martin, Shouten, \& Haxby, 1999; Aguirre, Zarahn, \& D'Esposito, 1998; Epstein \& Kanwisher, 1998), and that can be similarly activated by semantic processing of the same categories even in the absence of visual input (Chao, Haxby, \& Martin, 1999). These areas probably constitute the functional equivalent of monkey inferotemporal cortex regions that have been extensively investigated, demonstrating selectivity for complex visual features and object identity (Vogels, 1999; Booth \& Rolls, 1998; Rolls \& Tovee, 1995; Tanaka, 1993, 1996). Altogether, these studies suggest that the sources of the decision-related activity that we isolated here could be located in occipito-temporal regions.

\section{DISCUSSION}

We have shown and separated here two distinct processing stages in high-level visual categorization tasks. The first one corresponds to a "perceptual" stage and is found to reflect the category of the picture constituting the visual input as early as 75-80 msec. Other equivalently early differences correlated with high-level properties of the visual stimuli have been reported before (Mouchetant-Rostaing et al., 2000; George et al., 1997; Seeck et al., 1997). Yet it remained questionable how much of these activities could be attributed to concurrent low-level visual differences, changes in the subject's attentional state or in the experimental procedure between the different conditions compared. Here, with equally balanced conditions in terms of the relevance of the stimuli towards the task, and a systematic alternation between the two tasks, allowing comparison between trials that are temporally interleaved, we demonstrate that this early differential activity between visually defined categories is not correlated with high-level taskrelated properties. Therefore, it probably only reflects systematic visual differences in the "low-level" properties of the visual categories involved. More specifically, it is highly likely that this category-specific activity simply reflects visual encoding mechanisms and the extraction of numerous basic visual properties, the global energy of which can vary across categories. However, the difference demonstrated here should not be understood as a simple statistical difference between any two ensembles of images. For example, there is no such early differential activity when comparing the processing of vehicle pictures when they are targets and when they are nontargets: These two subsets were randomly extracted from the same original database.

The second mechanism that we found is correlated with the subject's behavior; it can also be seen when comparing the same visual category on trials where images are targets and on nontarget trials. Thus, this differential activity can be thought of as a correlate of 
the decision of the subject, regardless of the visual category that is to be treated as a target (i.e., of the visual details of the task).

It is important to note that subject reaction times were considerably shorter than those reported in a previous similar study (Thorpe et al., 1996). This is probably in large part due to changes in the experimental paradigm: The angular size of the images was larger, and we used a capacitive plate to detect subject responses rather than a mouse button release. The result was that median reaction times were about $100 \mathrm{msec}$ shorter (VanRullen \& Thorpe, in press). Despite this, the latency of the onset of the differential response at 150 msec was very similar to that reported previously, indicating that only the motor component of the task has been "compressed." The time required for target detection, on the other hand, appears fairly constant across these two studies. Further evidence for the fixed latency of the differential activity at $150 \mathrm{msec}$ comes from another recent study showing that even 3 weeks of intensive training failed to decrease this latency (FabreThorpe, Delorme, Marlot, \& Thorpe, 2001).

A direct consequence is that experiments reporting early "high-level" differential ERP activities in humans, or equivalently early differences in electrophysiological recordings in monkeys, should be treated with caution. A neuron or neuron assembly responding differently to different "categories" of visual input is not by itself evidence that sufficient visual processing has been done to allow categorization. To put it another way, what the experimenter reads out is not necessarily what the brain is actually reading out. As an example, if one was to "record" electrical activity in a computer while it is processing an integer variable to determine whether it is a prime number, one would find that the least significant bit of the binary-encoded integer is on average more "active" on prime than on nonprime numbers. However, it takes much more from the computer to decide if the variable is a prime number than to just check whether it is an odd or even integer. Before processing itself has even started, the preliminary encoding mechanisms can sometimes reflect, on average, high-level properties of the input variables. Comparing the average signal for trials presenting that property with trials that do not is clearly not sufficient to claim that the property has been extracted. A major advantage of dual-task paradigms such as the one used here is to allow the study of one particular high-level property independent of the underlying concurrent low-level properties.

Whether the two distinct activities shown here enter visual awareness or not is clearly not a straightforward question to answer, and probably beyond the scope of the present study. Also, much more attention has been devoted to the localization of visual awareness (Logothetis, 1998; Sahraie et al., 1997; Koch \& Braun, 1996a, 1996b; Leopold \& Logothetis, 1996; Crick \& Koch, 1995) than to its latency (Libet, Gleason, Wright, \& Pearl, 1983). A rather obvious conclusion is that the first perceptual activity, starting at around $75-80 \mathrm{msec}$, is not directly and immediately accessible to awareness; it would otherwise be correlated with the subject's behavior. Indeed, subjects could make use of that information to generate the motor command. In contrast, numerous theories suppose that neural activity at the final stages of the ventral "what" stream is correlated with the aware percept (Bar \& Biederman, 1999; Koch \& Braun, 1996a, 1996b; Crick \& Koch, 1995; Milner \& Goodale, 1995). Along with such views, the second, decision-related activity arising at $150 \mathrm{msec}$ could, directly or indirectly, participate in the awareness of the visual scene. The present data however is not sufficient to reject or to confirm such an interpretation.

\section{METHODS}

\section{Experimental Procedure}

Sixteen subjects, eight males and eight females with ages ranging from 21 to 50 were seated in a dark room, free of movement, at $120 \mathrm{~cm}$ from a computer screen piloted from a PC computer. Two categorization tasks with a go/ no-go paradigm were performed in alternation by all subjects. In each task, they viewed 10 series of 96 images, half of which were targets and half were nontargets. A trial was organized as follows: a white fixation point (smaller than $0.1^{\circ}$ of visual angle) was drawn in the middle of the black screen; subjects had to press a touch-sensitive button to start the trial; after a delay of $1 \mathrm{sec}$, the image that subtended roughly $10^{\circ}$ of visual angle in width and $7^{\circ}$ in height, was flashed at the center of the screen for $20 \mathrm{msec}$, and subjects had to release the button within $1 \mathrm{sec}$ if the image contained an element of the target category, and maintained pressure otherwise; the stimulus onset asynchrony (SOA) was 2 sec plus or minus a random delay of no more than 200 msec, to prevent the subjects from locking their response to the expected time of presentation. After the SOA, the subject's pressure on the touch-sensitive button was tested: The image was flashed only if the subject was ready, otherwise a delay of $1 \mathrm{sec}$ was added. Note that the very short presentation time ensures that there is no exploratory eye movement during image presentation.

\section{Images}

The pictures were natural photographs of complex scenes taken from a large commercially available CDROM library. The images in each category were chosen to be as varied as possible. The animal category included pictures of mammals as well as birds, fish, insects, or reptiles. The means of transport category included images of cars, trucks, trains, civil and military airplanes, helicopters, boats, hot air balloons, and even rockets. 
There was no a priori information on the size, position, or number of the targets in a single photograph. There was also a very wide range of distractor images, which could be outdoor or indoor scenes, natural landscapes or street scenes with buildings and roads, pictures of food, fruits, vegetables or plants, houses, man-made objects or tools. In each series, subjects were presented with 96 photographs, 48 targets and 48 nontargets. Half of the nontarget images were targets from the other task, i.e., vehicles in the animal task, and animals in the means of transport task. Furthermore, half of the vehicle images (24 per series in the means of transport task, 12 per series in the animal task) were images of cars and the other half of different means of transport, to allow further data analysis of intracategory differences. To prevent contamination by the effects of learning, an image was presented once for each subject and could not appear both as a target in one task and a nontarget in the other task. Image sequences were randomized so that different subjects were presented different image sequences over the same series of 96 trials, and that the presentation of a target was not predictable. Finally, all subjects alternately viewed 10 series of 96 trials for each task, and the order of appearance of the series was randomly decided for each subject, as well as whether they would start with an animal or a means of transport categorization series.

\section{ERP Recordings}

Electric brain potentials were recorded from 32 electrode sites equally distributed over the skull. Data acquisition was made at $1000 \mathrm{~Hz}$ (corresponding to a sample bin of 1 msec) using a Synamps recording system coupled with a PC computer. Recorded potentials were average-referenced on electrode CZ, and low-pass filtered at $100 \mathrm{~Hz}$. Trials were checked for artifacts and discarded using a $[-50 ;+50 \mu \mathrm{V}]$ criterion over the interval $[-100 ;+400$ msec] on frontal electrodes for eye movements and a $[-30 ;+30 \mu \mathrm{V}]$ criterion on the period $[-100 ;+100$ msec] on parietal electrodes for "alpha" brain waves. Electrodes were grouped into frontal (FP1, FP2, F3, F4, F7, F8, FZ in the 10-20 system nomenclature), central (C3, C4, CZ), parietal (PZ, PO3, PO4, POZ) and occipital groups (O1, O2, PO7, PO8, OZ) according to position, so as to represent the whole midline. Intersubjects statistical $t$ tests $(d f=15)$ were performed at the $p<.01$ level on the parietal electrodes group for the differential activities shown in Figure 3 and on frontal electrodes for the differential activities shown in Figure 4. According to the criterion of Rugg, Doyle, and Wells (1995) (see also Thorpe et al., 1996), a difference potential is considered significant if 15 consecutive $t$ test values are below the $p$ $<.05$ level. Here we strengthen the precision of this criterion by using $p<.01$. Times at which significant differences emerge according to this criterion are indicated in the text by the sign *.

\section{Acknowledgments}

Reprint requests should be sent to: Rufin VanRullen, Centre de Recherche Cerveau and Cognition, UMR 5549, 133 Rte de Narbonne, 31062 Toulouse Cedex, France. E-mail: rufin@cerco. ups-tlse.fr.

\section{REFERENCES}

Aguirre, G. K., Zarahn, E., \& D'Esposito, M. (1998). An area within human ventral cortex sensitive to "building" stimuli: Evidence and implications. Neuron, 21, 373-383.

Allison, T., Puce, A., Spencer, D. D., \& McCarthy, G. (1999). Electrophysiological studies of human face perception: I. Potentials generated in occipito-temporal cortex by face and non-face stimuli. Cerebral Cortex, 9, 415-430.

Bar, M., \& Biederman, I. (1999). Localizing the cortical region mediating visual awareness of object identity. Proceedings of the National Academy of Sciences, U.S.A., 96, 1790-1793.

Booth, M. C. A., \& Rolls, E. T. (1998). View-invariant representations of familiar objects by neurons in the inferior temporal visual cortex. Cerebral Cortex, 8, 510-523.

Bötzel, K., Schulze, S., \& Stodieck, S. R. (1995). Scalp topography and analysis of intracranial sources of face-evoked potentials. Experimental Brain Research, 104, 135-143.

Chao, L., Haxby, J. V., \& Martin, A. (1999). Attribute-based neural substrates in temporal cortex for perceiving and knowing about objects. Nature Neuroscience, 2, 913-919.

Crick, F., \& Koch, C. (1995). Are we aware of neural activity in primary visual cortex? Nature, 375, 121-123.

Debruille, J. B., Guillem, F., \& Renault, B. (1998). ERPs and chronometry of face recognition: Following-up Seeck et al. and George et al. NeuroReport, 9, 3349-3353.

Epstein, R., \& Kanwisher, N. (1998). A cortical representation of the local visual environment. Nature, 392, 598-601.

Fabre-Thorpe, M., Delorme, A., Marlot, C., \& Thorpe, S. (2001). A limit to the speed of processing in ultra-rapid visual categorization of novel natural scenes. Journal of Cognitive Neuroscience, 13(2), 171-180.

Fize, D., Boulanouar, K., Chatel, Y., Ranjeva, J. P., FabreThorpe, M., \& Thorpe, S. (2000). Brain areas involved in rapid categorization of natural images: An event-related fMRI study. Neuroimage, 11(6), 634-643.

George, N., Jemel, B., Fiori, N., \& Renault, B. (1997). Face and shape repetition effects in humans: A spatio-temporal ERP study. NeuroReport, 8, 1417-1423.

Halgren, E., Raij, T., Marinkovic, K., Jousmäki, V., \& Hari, R. (2000). Cognitive response profile of the human fusiform face area as determined by MEG. Cerebral Cortex, 10, 69-81.

Ishai, A., Ungerleider, L. G., Martin, A., Shouten, J. L., \& Haxby, J. V. (1999). Distributed representation of objects in the human ventral visual pathway. Proceedings of the National Academy of Sciences, U.S.A., 96, 9379-9384.

Jeffreys, D. A. (1996). Evoked potential studies of face and object processing. Visual Cognition, 3, 1-38.

Koch, C., \& Braun, J. (1996a). Towards the neuronal correlate of visual awareness. Current Opinion in Neurobiology, 6, 158-164.

Koch, C., \& Braun, J. (1996b). The functional anatomy of visual awareness. Cold Spring Harbor Symposia on Quantitative Biology, 61, 49-57.

Leopold, D. A., \& Logothetis, N. K. (1996). Activity changes in early visual cortex reflect monkeys' percepts during binocular rivalry. Nature, 379, 549-553.

Libet, B., Gleason, C. A., Wright, E. W., \& Pearl, D. K. (1983). Time of conscious intention to act in relation to onset of cerebral activity (readiness-potential). The unconscious initiation of a freely voluntary act. Brain, 106, 623-642. 
Logothetis, N. (1998). Object vision and visual awareness. Current Opinion in Neurobiology, 8, 536-544.

Luce, R. D. (1986). Response times. Oxford: Oxford University Press.

Milner, A. D., \& Goodale, M. A. (1995). The visual brain in action. Oxford: Oxford University Press.

Mouchetant-Rostaing, Y., Giard, M.-H., Bentin, S., Aguera, P. A., \& Pernier, J. (2000). Neurophysiological correlates of face gender processing in humans. European Journal of $\mathrm{Neu}$ roscience, 12, 303-310.

Newsome, W. T., Britten, K. H., \& Movshon, J. A. (1989). Neuronal correlates of a perceptual decision. Nature, 341, 52-54.

Nichols, M. J., \& Newsome, W. T. (1999). The neurobiology of cognition. Nature, 402, C35-C38.

Oram, M. W., \& Perrett, D. I. (1992). Time course of neural responses discriminating different views of the face and head. Journal of Neurophysiology, 68, 70-84.

Perrett, D. I., Rolls, E. T., \& Caan, W. (1982). Visual neurons responsive to faces in the monkey temporal cortex. Experimental Brain Research, 47, 329-342.

Rolls, E. T., \& Tovee, M. J. (1995). Sparseness of the neuronal representation of stimuli in the primate temporal visual cortex. Journal of Neurophysiology, 73, 713-726.

Romo, R., \& Salinas, E. (1999). Sensing and deciding in the somatosensory system. Current Opinion in Neurobiology, 9, 487-493.

Rossion, B., Gauthier, I., Tarr, M. J., Despland, P., Bruyer, R., Linotte, S., \& Crommelinck, M. (2000). The N170 occipitotemporal component is delayed and enhanced to inverted faces but not to inverted objects: An electrophysiological account of face-specific processes in the human brain. NeuroReport, 11, 69-74.

Rugg, M. D., Doyle, M. C., \& Wells, T. (1995). Word and non- word repetition within-and across-modality: An event-related potential study. Journal of Cognitive Neuroscience, 7, 209227.

Sahraie, A., Weiskrantz, L., Barbur, J. L., Simmons, A., Williams, S. C., \& Brammer, M. J. (1997). Pattern of neuronal activity associated with conscious and unconscious processing of visual signals. Proceedings of the National Academy of Sciences, U.S.A., 94(17), 9406-9411.

Schall, J. D., \& Thompson, K. G. (1999). Neural selection and control of visually guided eye movements. Annual Review of Neuroscience, 22, 241-259.

Schendan, H. E., Ganis, G., \& Kutas, M. (1998). Neurophysiological evidence for visual perceptual categorization of words and faces within $150 \mathrm{~ms}$. Psychophysiology, 35, 240251.

Seeck, M., Michel, C. M., Mainwaring, N., Cosgrove, R., Blume, H., Ives, J., Landis, T., \& Schomer, D. L. (1997). Evidence for rapid face recognition from human scalp and intracranial electrodes. NeuroReport, 8(12), 2749-2754.

Shadlen, M. N., \& Newsome, W. T. (1996). Motion perception: Seeing and deciding. Proceedings of the National Academy of Sciences, U.S.A., 93, 628-633.

Tanaka, K. (1993). Neuronal mechanisms of object recognition. Science, 262, 685-688.

Tanaka, K. (1996). Inferotemporal cortex and object vision. Annual Review of Neuroscience, 19, 109-139.

Thorpe, S. J., Fize, D., \& Marlot, C. (1996). Speed of processing in the human visual system. Nature, 381, 520-522.

VanRullen, R., \& Thorpe, S. J. (in press). Is it a bird? Is it a plane? Ultra-rapid visual categorisation of natural and artifactual objects. Perception.

Vogels, R. (1999). Categorization of complex visual images by rhesus monkeys: Part 2. Single-cell study. European Journal of Neuroscience, 11, 1239-1255. 